\title{
Etude des mousses de décontamination en écoulement : méthodes de caractérisation
}

\author{
The study of flow decontamination foams : characterization methods
}

par G. Boissonnet, B. Fournel, M. Faury

CEA Cadarache, DESD/SEP/LETD.

The use of foams for the decontamination of nuclear facilities has great potentialities. There is an increasing number of studies, concerning new foam formulations, adapted to new purposes. In order to sustain these actions, fundamental studies are in progress. Concerning foam flow, an Ostwald de Weale rheological behaviour has been found and constants of this model have been calculated. Foam structure and stability have also been studied using an image analysis method. Although foam structure can only be studied at the wall, this method is quite relevant, for it is a non-intrusive measurement. Yet, a little number of specific measuring devices have been developped at this time. Our present results encourage us to investigate new measuring methods in the particular domain of wet foams. This will enable us to improve rheological and stability modeling.

\section{I $\square$ INTRODUCTION}

\subsection{Contexte de l'étude}

Parmi les méthodes de décontamination des matériaux solides développées dans le nucléaire, à des fins de maintenance ou de démantèlement, l'emploi de mousses s'avère particulièrement intéressant pour la décontamination interne des composants de grand volume et de géométrie complexe. Une mousse est constituée d'une phase liquide continue et d'une phase gazeuse dispersée. Ainsi, l'utilisation de mousse permet de réduire d'un facteur 10 environ le volume des effluents produits par rapport aux techniques classiques utilisant des liquides. Le procédé a été validé à l'échelle industrielle lors de la décontamination d'un réfrigérant en 1993 [1]. Cette opération a permis de mettre en évidence des propriétés de mouillage très intéressantes grâce à la présence des tensioactifs. De plus, il a été constaté que la mousse remplit mieux les volumes et les interstices (faisceau de tubes, chicanes) des enceintes à décontaminer qu'un liquide. Les volumes morts sont plus faibles, ce qui assure un apport homogène des réactifs sur les parois. Enfin, dans certaines conditions opératoires, les mousses ont révélé des capacités d'érosion et de transport de particules nettement supérieures à celles des liquides. Cependant, les propriétés physiques d'une mousse en écoulement sont peu connues. Le but des travaux entrepris au Laboratoire d'Etudes des Techniques de Décontamination est d'acquérir une connaissance générale des propriétés et des caractéristiques d'une mousse de décontamination. Ces travaux visent également à développer et valider des méthodes de caractérisation spécifiques aux mousses.

1.2. Principe d'une décontamination par mousse et grandeurs physiques

Le principe de la décontamination peut être décrit par le schéma présenté sur la figure 1. La mousse (1) est utilisée comme un réservoir de réactifs. Un film liquide (2) ruisselle

1. Principe de la décontamination par mousse

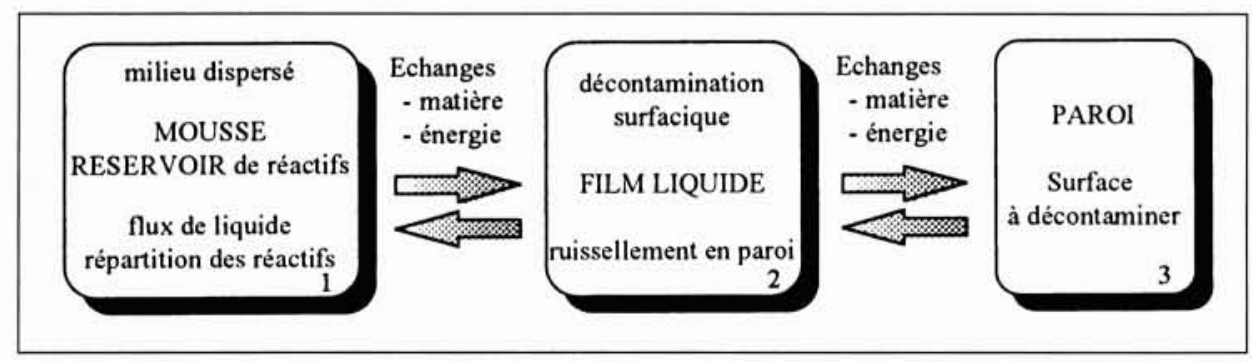


sur la paroi (3), assurant la décontamination. Des échanges de matières et d'énergie ont lieu entre ces trois éléments. La mousse est un système dynamique et thermodynamiquement instable doté de nombreux paramètres physiques et chimiques qu'il est difficile de relier entre eux. La connaissance de l'épaisseur du film et de sa composition, du comportement hydrodynamique de la mousse et de la nature des flux de liquide au sein de la mousse est nécessaire pour déterminer la répartition des réactifs dans le volume de mousse, dans le film et quantifier les échanges.

Deux études sont menées en parallèle. La première concerne le comportement hydrodynamique d'une mousse s'écoulant à une vitesse variant de 0,1 à $1,2 \mathrm{~m} \cdot \mathrm{s}^{-1}$ (temps de passage de 30 secondes à 6 minutes). La fraction massique de liquide est alors à peu près constante entre l'entrée et la sortie de l'enceinte. La seconde est consacrée à l'étude de la stabilité et de la structure des mousses. Dans ce cas, les vitesses d'écoulement sont de quelques millimètres par seconde (temps de passage de 10 à 20 minutes), ce qui permet de favoriser le ruissellement du liquide au cœur de la mousse. L'objectif final est de relier les résultats obtenus dans chacune des études et de construire différents modèles représentatifs des cas rencontrés industriellement.

\section{CARACTÉRISATION DU COMPORTE- MENT HYDRODYNAMIQUE}

\subsection{Etat des recherches}

\subsubsection{Propriétés rhéologiques}

Il existe deux types principaux d'appareillage pour mesurer les caractéristiques rhéologiques d'une mousse : le rhéomètre rotatif à régime permanent du type Couette et le rhéomètre tubulaire à régime permanent du type Poiseuille. L'utilisation d'un rhéomètre rotatif ne semble pas satisfaisante dans le cas d'une mousse. La dégradation de la mousse au cours de la mesure, l'influence de l'appareillage sur la structure de la mousse, la vitesse de glissement non nulle à la paroi du rhéomètre rendent impossible l'établissement d'un régime permanent lors de la mesure et sont cause d'un manque de reproductibilité.

En revanche, plusieurs facteurs plaident en faveur de l'utilisation de conduites cylindriques pour mesurer la contrainte et la vitesse de cisaillement. Dans ce cas la mousse s'écoule effectivement au lieu d'être uniquement cisaillée, situation qui s'apparente davantage aux conditions de sollicitations subies par une mousse dans un environnement industriel. De plus, contrairement à un rhéomètre rotatif, la mesure est effectuée en continu. Elle peut donc être poursuivie plus longtemps à partir de l'établissement d'un régime permanent.

\subsubsection{Vitesse de glissement}

Les études rhéologiques de fluides classiques s'appuient sur l'existence d'une couche limite d'écoulement proche de la paroi, dont l'existence permet de formuler une hypothèse de non glissement. Dans le cas des mousses, la majorité des auteurs ont constaté la présence en paroi d'un film liquide, sur lequel la mousse semble glisser. Thondavadi et al. [2] nuancent cette observation. Ils ont montré que pour une conduite en matériau synthétique, la mousse glisse sur un film liquide, en revanche, pour une conduite métallique l'écoulement se fait sans présence d'un film de glissement. Le débit total observé $\mathrm{Q}_{\mathrm{obb}}$, le débit dû au glissement $\mathrm{Q}_{\mathrm{gliss}}$ et le débit dû au cisaillement $Q_{\text {cisaill }}$ sont reliés ainsi :

$$
\mathrm{Q}_{\text {obs }}=\mathrm{Q}_{\text {gliss }}+\mathrm{Q}_{\text {cisaill }}
$$

En écoulement tubulaire, deux méthodes principales reposant sur le même principe ont été mises au point, l'une par Mooney [3] et l'autre par Olroyd-Jastrzebski [4]. Le principe de calcul consiste à mesurer l'évolution de la vitesse de cisaillement observée en fonction du diamètre $\mathrm{D}$ de la conduite, à contrainte de cisaillement en paroi $\tau_{\mathrm{p}}$ fixée, en traçant $\dot{\gamma}_{o b s}=\left(\frac{8 u}{D}\right)_{o b s}=\left(\frac{1}{D^{m}}\right)^{\text {(u vitesse linéaire moyenne, } \mathrm{m}}$ nombre entier fixé). La méthode de Mooney s'effectue avec $\mathrm{m}=1$ et d'Olroyd et Jastrzebski avec $\mathrm{m}=2$. La pente de la droite obtenue par régression linéaire est en relation directe avec la vitesse de glissement. Winkler et al. [4] ont testé les deux méthodes de mesure de la vitesse de glissement et de correction. Selon leurs résultats, la méthode de Mooney n'est pas appropriée aux mousses, la méthode Olroyd-Jastrzebski semble la mieux adaptée.

\subsubsection{Caractéristiques du film de glissement}

Le calcul de l'épaisseur du film $\delta$ est plus délicat que celui de la vitesse de glissement et sa mesure directe, n'est pas réalisable avec les techniques de mesure connues actuelle-

Tableau 1. - Méthodes d'estimation de l'épaisseur de la couche de glissement

\begin{tabular}{|l|l|l|c|l|l|}
\hline Auteur & Estimation & Hypothèse de travail & Valeur de $\delta$ & Relation entre : & Remarque \\
\hline Kraynik [5] & directe & $\begin{array}{l}\text { débit assuré à } 100 \% \\
\text { par le glissement }\end{array}$ & 2 à $4 \mu \mathrm{m}$ & $\begin{array}{l}\text { perte de pression } \\
\text { et } \delta\end{array}$ & $\begin{array}{l}\text { couche laminaire calcul à } \\
\text { foisonnement 20 }\end{array}$ \\
\hline Kroezen [6] & directe & $\begin{array}{l}\text { débit assuré à 100\% } \\
\text { par le glissement }\end{array}$ & 0,5 à $30 \mu \mathrm{m}$ & $\begin{array}{l}\text { perte de pression } \\
\text { et } \delta\end{array}$ & $\begin{array}{l}\text { comparaison entre } \delta \text { et } \\
\delta_{\text {b' }} \text { épaisseur de film } \\
\text { interbulle }\end{array}$ \\
\hline Kraynik [7] & directe & $\begin{array}{l}\text { épaisseur de la couche } \\
\text { de glissement liée au } \\
\text { diamètre des bulles }\end{array}$ & $10 \mu \mathrm{m}$ & $\begin{array}{l}\text { grandeurs physico- } \\
\text { chimiques, d (diamètre } \\
\text { moyen des bulles) et } \delta\end{array}$ & $\begin{array}{l}\text { fondée sur la quantité de } \\
\text { liquide dans les films } \\
\text { interbulles }\end{array}$ \\
\hline Hirasaki [8] & directe & $\begin{array}{l}\text { périmètre mouillé de la } \\
\text { couche liquide source } \\
\text { de la résistance à } \\
\text { l'écoulement }\end{array}$ & $10 \mu \mathrm{m}$ & $\begin{array}{l}\text { grandeurs physico- } \\
\text { chimiques, d et } \delta\end{array}$ & $\begin{array}{l}\text { fondée sur l'expression } \\
\text { 'une contrainte critique } \\
\text { d et d'un nombre de } \\
\text { capillarité }\end{array}$ \\
\hline Calvert [9] & indirecte & $\begin{array}{l}\text { débit assuré par le } \\
\text { glissement et par le } \\
\text { cisaillement }\end{array}$ & $20 \mu \mathrm{m}$ & $\begin{array}{l}\tau_{p^{\prime}} \dot{\gamma} \text { et } \delta \\
\text { couche laminaire } \\
\text { paramètre ajustable } \\
\text { du modèle d'écoulement }\end{array}$ \\
\hline
\end{tabular}


ment. Les valeurs sont obtenues à partir de modèles approchés, rassemblés dans le tableau 1.

L'ordre de grandeur de l'épaisseur du film de glissement varie de 2 à $30 \mu \mathrm{m}$ selon les travaux. Notons que cette valeur n'a pas toujours été obtenue dans des conditions expérimentales identiques, ce qui peut expliquer les variations observées.

\subsection{Etudes menées au CEA}

\subsubsection{Dispositif expérimental}

Le dispositif expérimental est composé d'une conduite cylindrique d'une longueur $\mathrm{L}=32$ mètres et d'un diamètre $\mathrm{D}=30$ millimètres. Ainsi la conduite est assez longue pour observer les effets de la compressibilité et son diamètre est assez grand pour limiter les effets de bords. La mousse est produite par un générateur statique garni d'un lit de billes de verre. La longueur du générateur et le diamètre des billes sont variables. Le gaz et la solution moussante sont injectés sous pression dans le générateur à travers le lit de billes, ce qui assure le mélange intime des deux phases. La conduite est jalonnée de quatre capteurs qui permettent de mesurer les pertes de pression engendrées par la circulation du fluide. Ainsi, la mousse s'écoule dans trois tronçons successifs. La qualité de la mousse est contrôlée à la sortie de la conduite par une prise d'échantillon. L'alimentation en liquide se fait par l'intermédiaire d'une pompe à engrenage pour assurer un débit stable. Le débit de liquide $Q_{1}$ et le débit de gaz dans les conditions normales de température et de pression $\mathrm{Q}_{\mathrm{g}}{ }^{0}$ sont mesurés respectivement par un débitmètre électromăgnétique et un débitmètre régulateur thermique. $\mathrm{Q}_{\mathrm{g}}$ est le débit de gaz à la pression $\mathrm{P}$ et à la température $\mathrm{T}$. Il est calculé à partir de $\mathrm{Q}_{\mathrm{g}}{ }^{0}$ en supposant que le gaz suit la loi des gaz parfaits. Le foisonnement $\mathrm{F}$ est le rapport entre le débit de mousse et le débit de liquide $Q_{1} \cdot Q_{m}{ }^{0}$ est le débit de mousse calculé dans les conditions normales de température et de pression, $\mathrm{Q}_{\mathrm{m}}$ est le débit de mousse à la pression $\mathrm{P}$ et à la température $\mathrm{T}$. $\mathrm{F}^{0}$ et $\mathrm{F}$ sont respectivement définis par :

$F^{0}=\frac{Q_{m}^{0}}{Q_{l}} \approx \frac{Q_{g}^{0}+Q_{l}}{Q_{l}} \quad$ (2) et $\quad F=\frac{Q_{m}}{Q_{l}} \approx \frac{Q_{g}+Q_{l}}{Q_{l}}$

Un essai expérimental s'effectue à fraction massique de liquide constante c'est-à-dire pour une valeur de $F^{0}$ fixée.

\subsubsection{Définition d'un modèle d'écoulement adapté aux mousses}

Modèles classiques. La mousse étant un fluide diphasique, il est naturel de penser que des modèles d'écoulement de fluides diphasiques gaz-liquide ou d'émulsions peuvent être adaptés. Mais Calvert et al. [10] ont montré que l'utilisation de tels modèles conduit à des pertes de pression calculées 10 à 1000 fois inférieures à celles mesurées expérimentalement. De fait, la mousse est assimilée à un fluide homogène quant à son écoulement par la plupart des auteurs. Ce choix repose sur le fait que la taille du contenant est beaucoup plus grande que celle des bulles. Heller et al. [7] proposent de considérer que le fluide est homogène, quand le diamètre de la conduite est supérieur à 20 fois la taille maximale des bulles. De plus, Calvert et al. [9] avancent que le gaz emprisonné dans la matrice liquide s'écoule à la même vitesse que celle-ci. Les trois modèles, les plus couramment utilisés pour caractériser les mousses, reliant la contrainte de cisaillement $\tau$ et la vitesse de cisaillement $\dot{\gamma}$, sont ceux de Bingham, d'Ostwald de Waele, et d'Herschel-Bulkley.

Compressibilité de la mousse. Les courbes reportées sur la figure 2 représentent la contrainte en paroi $\tau=\mathrm{D} \Delta \mathrm{P} / 4 \mathrm{~L}$ en fonction de la vitesse de cisaillement $\dot{\gamma}=8 \mathrm{u} / \mathrm{D}$ pour trois tronçons successifs de la conduite. $\Delta \mathrm{P}$ est la perte de pres- sion, D est le diamètre de la conduite, $\mathrm{L}$ est la longueur du tronçon et $u$ est la vitesse moyenne de circulation du fluide. Dans le cas d'un fluide incompressible, la grandeur $\tau$ serait constante le long de la conduite à débit fixé, ainsi les trois courbes seraient confondues. La compressibilité de la mousse entraîne une augmentation du débit volumique due à l'expansion du fluide et une augmentation des pertes de pression linéiques le long de la conduite. De même, la figure 3 regroupant les courbes de perte de pression totale sur l'ensemble du circuit pour différents foisonnements $\mathrm{F}^{0}$, montre que la perte de pression augmente quand le foisonnement augmente. Ces deux observations montrent que la fraction volumique de gaz joue un rôle fondamental dans le comportement hydrodynamique de la mousse et que la compressibilité ne peut pas être négligée. Il est donc nécessaire d'établir un modèle tenant compte de ces deux grandeurs.

Modèle d'écoulement spécifique aux mousses. Dans le cas d'un fluide incompressible ou compressible et newtonien, la relation entre le facteur de frottement et le nombre de Reynolds s'écrit : $\frac{f}{2}=\frac{8}{R e}$ en régime laminaire. Cette relation

n'est plus valable dans le cas d'un fluide compressible non newtonien. Le facteur de frottement n'est donc pas a priori constant le long de la conduite et il est nécessaire de calculer un facteur de frottement local. Dans le cas d'un fluide com-

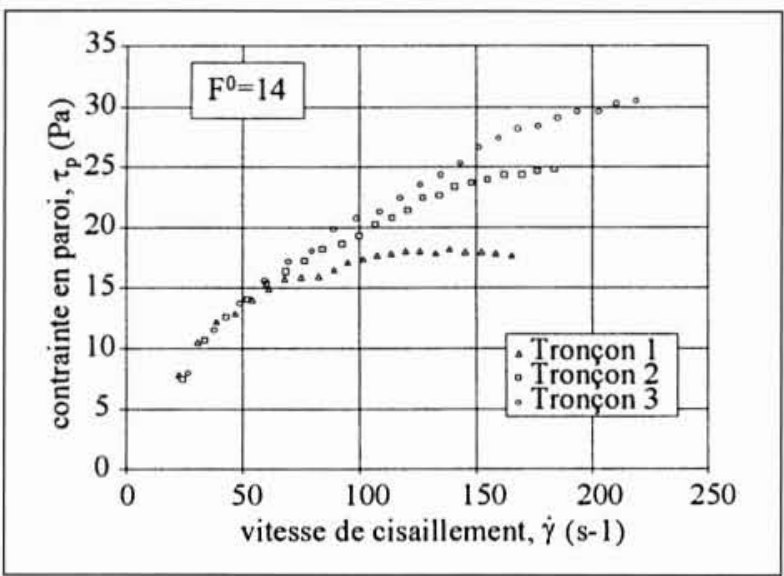

2. Pertes de pression linéiques $\Delta \mathrm{P} / \mathrm{L}$ en fonction du débit $\mathrm{Qm}^{0}$ à foisonnement $\mathrm{F}^{0}=14$

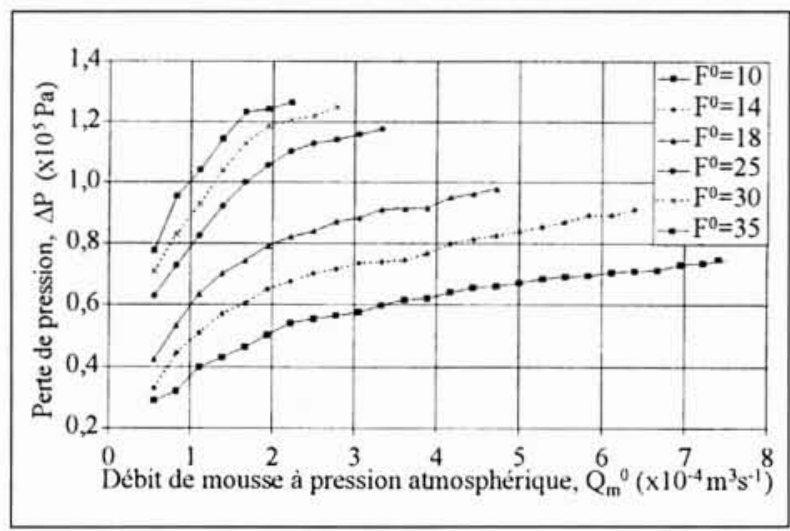

3. Pertes de pression totale sur l'ensemble du circuit en fonction du débit $\mathbf{Q m}^{0}$ pour différentes valeurs de foisonnement $\mathrm{F}^{0}$ 
pressible non newtonien s'écoulant dans une conduite de diamètre $\mathrm{D}$ constant, à température constante et en régime laminaire, il est intéressant de se ramener à un état de référence noté * pour lequel le facteur de frottement est constant le long de la conduite. En particulier, $f$ n'est pas lié à la pression dans la conduite, ni à ses variations. Dans une étude consacrée à l'écoulement de mousses de forage, destinées à l'injection dans des failles géologiques, Valko et al. [11] ont défini un modèle fondé sur le principe nommé « volume equalized principle ». Ce modèle consiste à étudier le fluide grâce aux variables réduites $\dot{\gamma}^{*}=\dot{\gamma} / \varepsilon$ et $\tau_{p}^{*}=\tau_{p} / \varepsilon$, pour

lesquelles $\varepsilon$ représente le facteur d'expansion spécifique $\varepsilon=\rho * / \rho, \rho$ étant la masse volumique de la mousse et $\rho^{*}$ la masse volumique liquide constituant la mousse.

Au cours de nos travaux, nous formulons l'hypothèse que pour la mousse que nous étudions, les variables réduites permettent de travailler comme si le fluide était incompressible.

\subsubsection{Résultats obtenus sur la base du modèle : véri- fication de l'hypothèse et ajustement}

La figure 4 représente un rhéogramme tracé, dans le domaine de référence, pour trois valeurs de foisonnement $\mathrm{F}^{0}$. La reproductibilité des mesures est meilleure que celle donnée par Calvert et al. [10]. En effet, la dispersion des mesures expérimentales est d'environ $5 \%$ au lieu de $20 \%$. Cette courbe montre que $\dot{\gamma}^{*}$ et $\tau_{\mathrm{p}}{ }^{*}$ sont liés par une relation bijective indépendamment du foisonnement, dans le domaine $10<\mathrm{F}^{0}<18$. L'hypothèse que nous avons formulée au paragraphe précédent concernant la mousse est donc vérifiée.

Une légère dérive est observable pour des valeurs expérimentales correspondant aux débits les plus forts et donc aux pressions les plus élevées. La valeur du foisonnement réel $\mathrm{F}$ est alors d'environ 5 à 6 . Calvert et al. [12] signalent qu'autour de cette valeur, la structure de la mousse change, ce qui pourrait entraîner une modification de son comportement hydrodynamique. Nous avons donc choisi de concentrer notre étude sur la partie linéaire des courbes. Le modèle d'écoulement a été identifié comme étant de type Ostwald de Weale et les valeurs des paramètres du modèle ont été calculées par régression linéaire. L'équation rhéologique obtenue s'écrit :

$$
\tau_{p}^{*}=0.45\left(\dot{\gamma}^{*}\right)^{0.6}
$$

Nous constatons l'absence de contrainte critique de cisaillement qui pourrait s'expliquer par la présence du film de glissement en paroi, dont l'existence reste à confirmer. Les caractéristiques rhéologiques étant connues, il est possible de déterminer une relation entre le facteur de frottement théorique et le nombre de Reynolds.

$$
\frac{f}{2}=\frac{6 n+2}{n} \cdot \frac{1}{R e} \quad \text { (soit avec } \mathrm{n}=0,6, \frac{f}{2}=\frac{9.33}{R e} \text { ) }
$$

\subsection{Conclusion}

Le modèle présenté ici permet de caractériser l'écoulement d'une mousse de décontamination et de prévoir les besoins énergétiques nécessaires pour sa circulation. Le dispositif de mesure utilisé donne des résultats fiables et reproductibles. Cependant, à lui seul, il ne permet pas d'accéder à l'épaisseur du film et à la vitesse de glissement. Des essais réalisés avec des conduites de diamètres variables sont en cours pour évaluer la vitesse de glissement et estimer l'épaisseur du film. Les besoins spécifiques de ce type d'études en matière de méthodes de mesures seront détaillés dans le paragraphe 4 .

\section{III ם STABILITÉ, DRAINAGE, STRUCTURE}

Dans le cas d'une opération de décontamination d'une enceinte de grand volume, le temps de séjour de la mousse est de l'ordre de 10 à 20 minutes. Sous l'effet de la gravité, le liquide interstitiel présent entre les bulles ruisselle dans le lit de mousse et le long des parois ; ce phénomène est appelé drainage. La mousse est ici étudiée sous son aspect de fluide dispersé. La décontamination intervenant à l'interface liquide/solide, il est nécessaire de caractériser le film en paroi, quant à son épaisseur, sa composition et sa vitesse d'écoulement. Les mesures directes n'ont pas été développées à ce jour ; cependant, les caractéristiques du film sont liées à la structure de la mousse. Il est donc intéressant d'étudier cette structure et particulièrement la distribution des tailles de bulles.

\subsection{Etat des recherches}

\subsubsection{Etude de la structure d'une mousse}

Deux méthodes principales ont été utilisées à ce jour, les méthodes photographiques présentées dans le tableau 3 et les méthodes optiques.

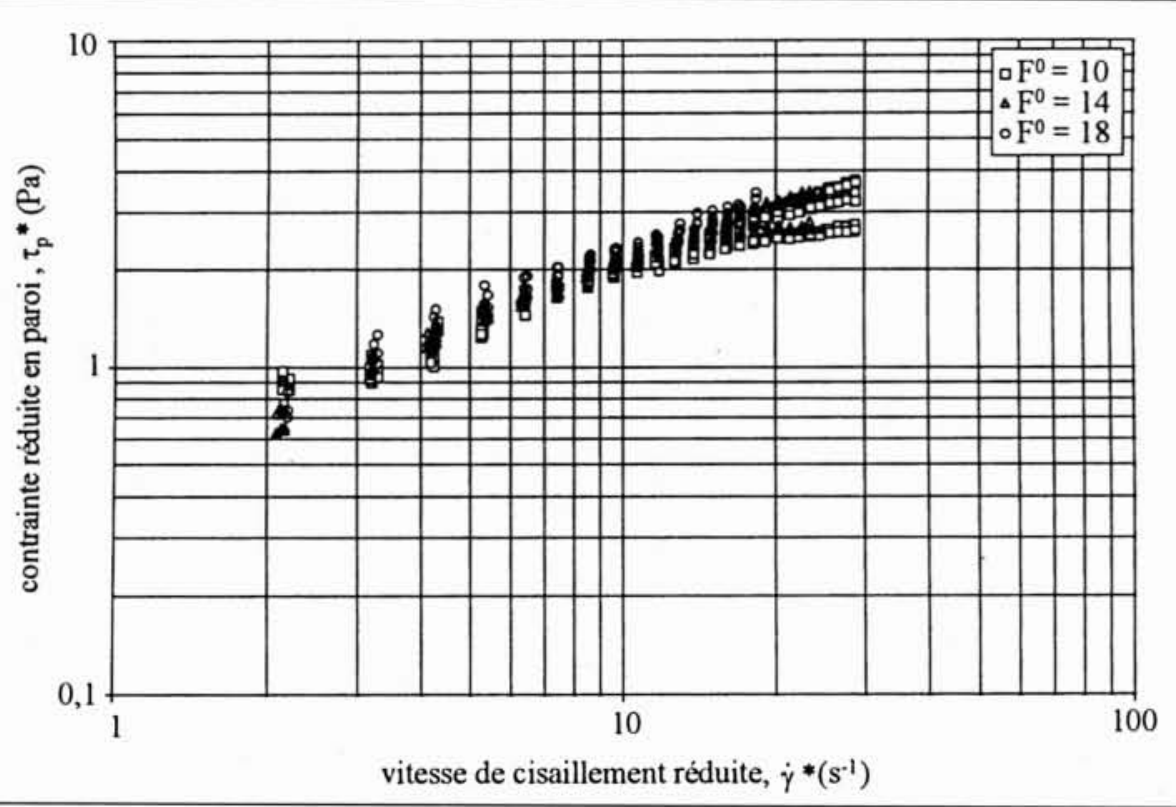


Tableau 3. - Méthodes de mesure photographique

\begin{tabular}{|c|l|l|l|}
\hline \multicolumn{1}{|c|}{ Auteur } & \multicolumn{1}{|c|}{ Dispositif } & Méthode de traitement & \multicolumn{1}{c|}{ Remarque } \\
\hline Cheng [13] (1983) & appareil photographique & $\begin{array}{l}\text { tirage papier et } \\
\text { mesure planimétrique }\end{array}$ & $\begin{array}{l}\text { génération de la mousse bulle par bulle } \\
\text { (4 tailles) mousse en écoulement (colonne) }\end{array}$ \\
\hline $\begin{array}{c}\text { Kroezen [14] } \\
\text { (1987) }\end{array}$ & $\begin{array}{l}\text { microscope + appareil } \\
\text { photographique }\end{array}$ & - & $\begin{array}{l}\text { construction des distributions par mesure } \\
\text { du diamètre de chaque bulle }\end{array}$ \\
\hline $\begin{array}{c}\text { Calvert [12] } \\
\text { (1987) }\end{array}$ & microscope + caméra CCD & $\begin{array}{l}\text { mesure manuelle et } \\
\text { traitement semi-au- } \\
\text { tomatique }\end{array}$ & $\begin{array}{l}\text { mousse en écoulement (conduite cylindrique) } \\
\text { construction des distributions par mesure } \\
\text { du diamètre de chaque bulle (100 bulles) }\end{array}$ \\
\hline $\begin{array}{c}\text { Gido [15] } \\
\text { (1989) }\end{array}$ & $\begin{array}{l}\text { microscope + appareil } \\
\text { photographique }\end{array}$ & mesure manuelle & $\begin{array}{l}\text { mousse en écoulement (cellule de visuali- } \\
\text { sation) construction des distributions par } \\
\text { mesure du diamètre de chaque bulle }\end{array}$ \\
\hline $\begin{array}{c}\text { Guillerme [16] } \\
\text { (1993) }\end{array}$ & caméra CCD numérique & $\begin{array}{l}\text { traitement et d'analyse } \\
\text { d'image automatique }\end{array}$ & $\begin{array}{l}\text { mousse en écoulement (colonne) } \\
\text { information globale (texture de la mousse) } \\
\text { obtenue par superposition de caches }\end{array}$ \\
\hline
\end{tabular}

La plupart des méthodes optiques présentent l'inconvénient majeur d'être intrusives ou réalisées sur des échantillons de mousses statiques. Au contraire, les méthodes photographiques évitent l'interaction entre la mousse et le dispositif de mesure et les mesures sont réalisables en continu. Jusqu'à présent, l'acquisition et l'analyse des images limitait l'utilisation de ce type de méthode, mais il est aujourd'hui possible acquérir jusqu'à 25 images par seconde avec un dispositif classique et plus pour des besoins spécifiques.

Quelques erreurs de mesure ont été mises en évidence [13] car la mesure est réalisée en paroi, mais en l'absence de méthode de référence, la mesure par photographie est très utile, d'autant plus que l'information en paroi est primordiale dans le cadre d'une décontamination surfacique. C'est la raison pour laquelle nous avons retenu cette méthode.

\subsubsection{Modèles de drainage}

Quelques modèles de drainage des mousses existent. Un modèle développé par Verbist et al. [17] représente la mousse par un réseau de canaux renfermant le liquide. L'équation générale est :

$$
\frac{\partial \alpha}{\partial \tau}+\frac{\partial}{\partial \xi}\left(\alpha^{2}-\frac{\sqrt{ } \alpha}{2} \frac{\partial \alpha}{\partial \xi}\right)=0
$$

où $\xi$, $\tau$ et $\alpha$ sont des grandeurs adimensionnelles correspondant à la position verticale mesurée du haut vers le bas $\mathrm{x}$, au temps t, et à la section des canaux $\mathrm{A}$. Elles sont définies par les relations $\mathrm{x}=\xi \mathrm{x}_{0}, \mathrm{t}=\tau \mathrm{t}_{0}$ et $\mathrm{A}=\alpha \mathrm{x}_{0}{ }^{2}$, où $x_{0}=\sqrt{C \sigma / \rho g}$ et $t_{0}=\eta^{*} / \sqrt{C \sigma \rho g}$

Les grandeurs physiques sont $\sigma$ : tension superficielle, $\rho$ : masse volumique du liquide, $\mathrm{g}$ : accélération de la gravité et $\eta^{*}$ : viscosité effective dont la signification ne sera pas discutée ici. $C=\sqrt{\sqrt{3}-\pi / 2}$ est une constante calculée en

considérant que les canaux sont triangulaires. Bien que certaines hypothèses soient restrictives, ce modèle a été validé expérimentalement pour des mousses statiques et sèches. Nos travaux visent à adapter ce modèle à une mousse en écoulement dont la fraction gazeuse serait comprise entre 0,8 et 0,9 . Un couplage entre le modèle de Verbist et al. [17] et un modèle d'écoulement dans un milieu poreux suivant la loi de Darcy pourrait également conduire à des résultats intéressants.

\subsection{Etudes menées au CEA}

\subsubsection{Dispositif expérimental}

Le dispositif expérimental (voir figure 5) est composé d'une colonne octogonale, de 30 litres et 1 mètre de haut. La mousse est générée par brassage du gaz et du liquide passant à travers un lit de billes comme dans le cas de l'étude rhéologique $(\S 2)$. Le débit de liquide $\mathrm{Q}_{1}$ et le débit de gaz $\mathrm{Q}_{\mathrm{g}}{ }^{0}$ sont mesurés respectivement par un débitmètre magnétique et un débitmètre régulateur thermique. Ainsi, le foisonnement $\mathrm{F}^{0}$ est connu à l'entrée de la colonne. La mousse s'écoule verticalement vers le haut dans la colonne : son débit est compris entre $4.10^{-5}$ et $9.10^{-5} \mathrm{~m}^{3} \mathrm{~s}^{-1}$, soit une vitesse linéaire ascendante d'écoulement de $1,3.10^{-3} \mathrm{~ms}^{-1}$ à $2,9.10^{-3}$ $\mathrm{ms}^{-1}$. Par souci de similitude avec les opérations industrielles, la mousse sortant en haut de la colonne est recyclée dans le réacteur contenant la solution moussante et dégradée immédiatement par aspersion d'alcool méthyl-4 pentanol-2. Il est ainsi possible de travailler en circuit fermé pendant 3 à 4 heures, la stabilité de la mousse étant ensuite limitée par l'accumulation d'alcool.

Au cours d'un essai, le liquide de drainage récupéré en fond de colonne est réintroduit en continu dans le réacteur par l'intermédiaire d'une pompe à engrenage et son débit est

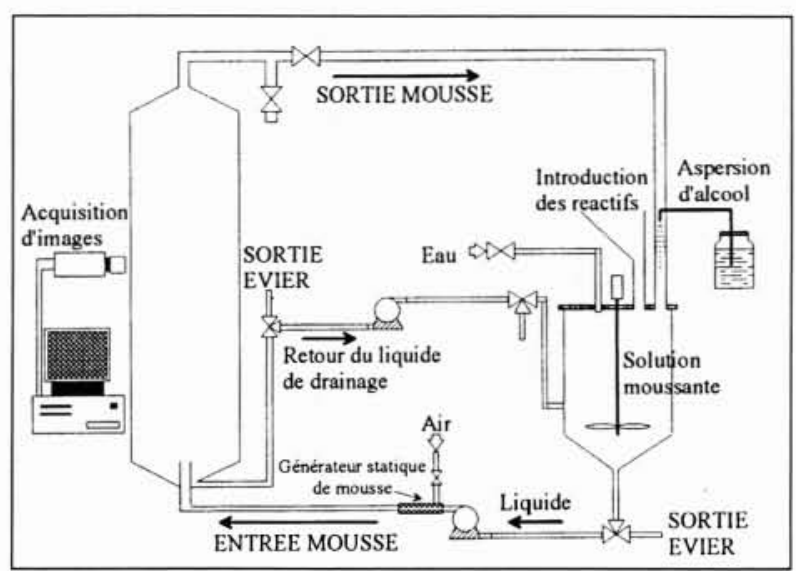

5. Dispositif d'étude de la stabilité et de la structure d'une mousse en écoulement 
mesuré. Un bilan des débits entrée-sortie permet de calculer le foisonnement en haut de la colonne $\mathrm{F}_{\text {bilan }}$. Un prélèvement d'échantillon de mousse permet de mesurer le foisonnement en haut de la colonne $\mathrm{F}_{\text {sortie }}$. L'écoulement de la mousse est en régime permanent quand $\mathrm{F}_{\text {bilan }}=\mathrm{F}_{\text {sortie }}$. Le temps de mise en régime varie de 5 minutes à 3 heures selon la valeur du débit de mousse $\mathrm{Q}_{\mathrm{m}}{ }^{0}$ et le foisonnement $\mathrm{F}^{0}$.

\subsubsection{Acquisition, Traitement et analyse d'image}

Pour réaliser les mesures, nous disposons d'une caméra $\mathrm{CCD}$ numérique couplée à un ordinateur. Le dispositif permet l'acquisition en temps réel de 7 images par seconde (figure 6a). Un traitement d'images consiste à isoler les informations significatives comme le contour des bulles, en contrastant l'image grâce à des filtres linéaires et non linéaires. L'image binaire obtenue après le seuillage (figure 6b) est nettoyée et les bulles sont remplies et répertoriées (figure $6 \mathrm{c}$ ), une couleur correspondant à un numéro. Les mesures peuvent alors être réalisées.

3.2.3. Résultats expérimentaux : distribution des tailles de bulles et diamètre moyen

La distribution des tailles de bulles représentée sur la figure 6d a été construite avec 10 images correspondant environ à 700 bulles ne touchant pas les bords de l'image. L'allure des différentes distributions construites pour plusieurs conditions opératoires est identique. En première approximation, la distribution des tailles de bulles est log-normal, ce qui est en accord avec d'autres études $[12,18]$. Il pourrait également s'agir d'une distribution exponentielle de l'aire des bulles.
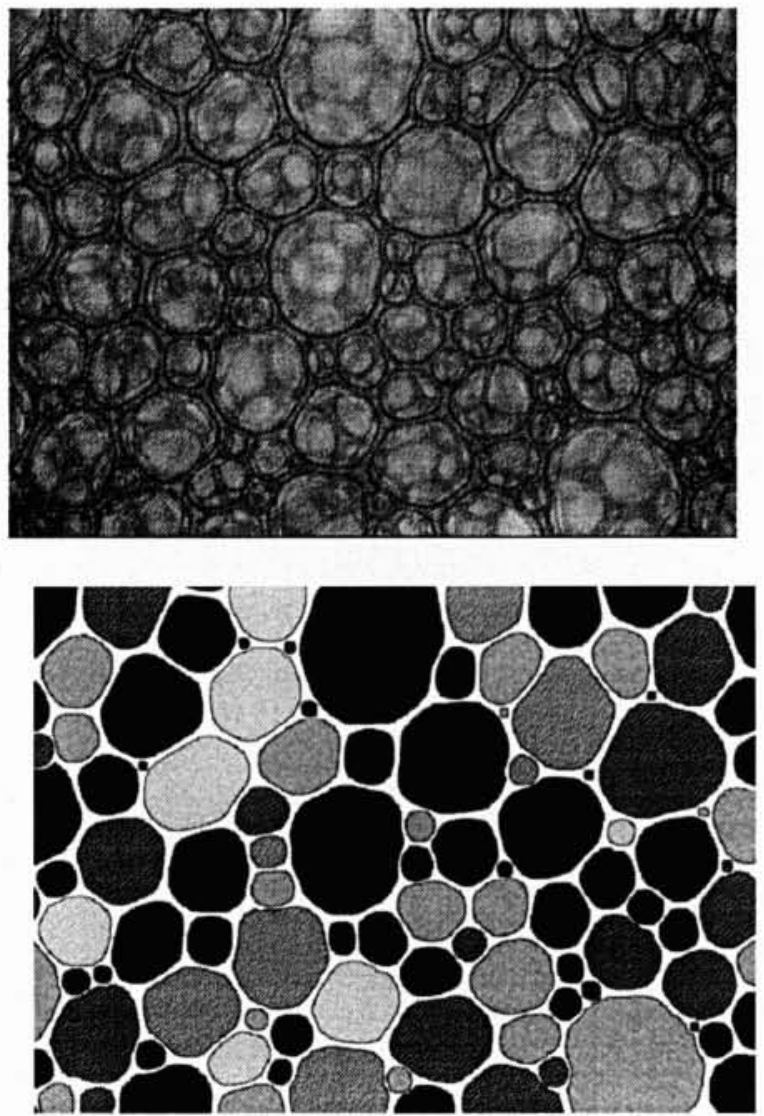

Des recherches bibliographiques sont en cours pour étayer cette hypothèse.

L'étude du diamètre moyen en paroi a révélé une variation d'environ $5 \%$ entre l'entrée et la sortie de la còlonne et dans les mêmes proportions quand le débit varie de $4.10^{-5}$ et $9.10^{-5} \mathrm{~m}^{3} \mathrm{~s}^{-1}$. Cette valeur est faible par rapport à la variation du foisonnement dans les mêmes conditions. Cette observation entraîne l'hypothèse suivante: l'influence des phénomènes de parois sur la structure externe de la mousse pourrait prédominer sur celle des phénomènes volumiques, tels que la génération ou le débit de mousse.

\subsubsection{Etude du foisonnement - Premières relations}

avec le drainage et la stabilité.

Sur la figure 7 sont représentés le foisonnement volumique $\mathrm{F}_{3 \mathrm{D}}$ mesuré grâce à une prise d'échantillon et le foisonnement mesuré sur la paroi grâce aux images $\mathrm{F}_{2 \mathrm{D}}$. On peut noter que :

- la valeur du foisonnement augmente quand la hauteur d'observation augmente,

- pour une hauteur d'observation fixée, le foisonnement diminue quand le débit de mousse augmente.

Ces observations sont à relier au fait que plus le temps de passage est long (débit faible), plus les effets du drainage sont importants.

La comparaison des foisonnements, permet de constater que les courbes de $\mathrm{F}_{2 \mathrm{D}}$ et $\mathrm{F}_{3 \mathrm{D}}$ ont la même allure et que $\mathrm{F}_{3 \mathrm{D}}$ est plus grand que $\mathrm{F}_{2 \mathrm{D}}$. De plus, $\mathrm{F}_{3 \mathrm{D}}$ augmente dans un rapport maximum de 5 entre l'entrée et la sortie de la colonne alors que $F_{2 D}$ n'augmente que dans un rapport de 2 . Cette observation semble confirmer le fait que les phénomènes de
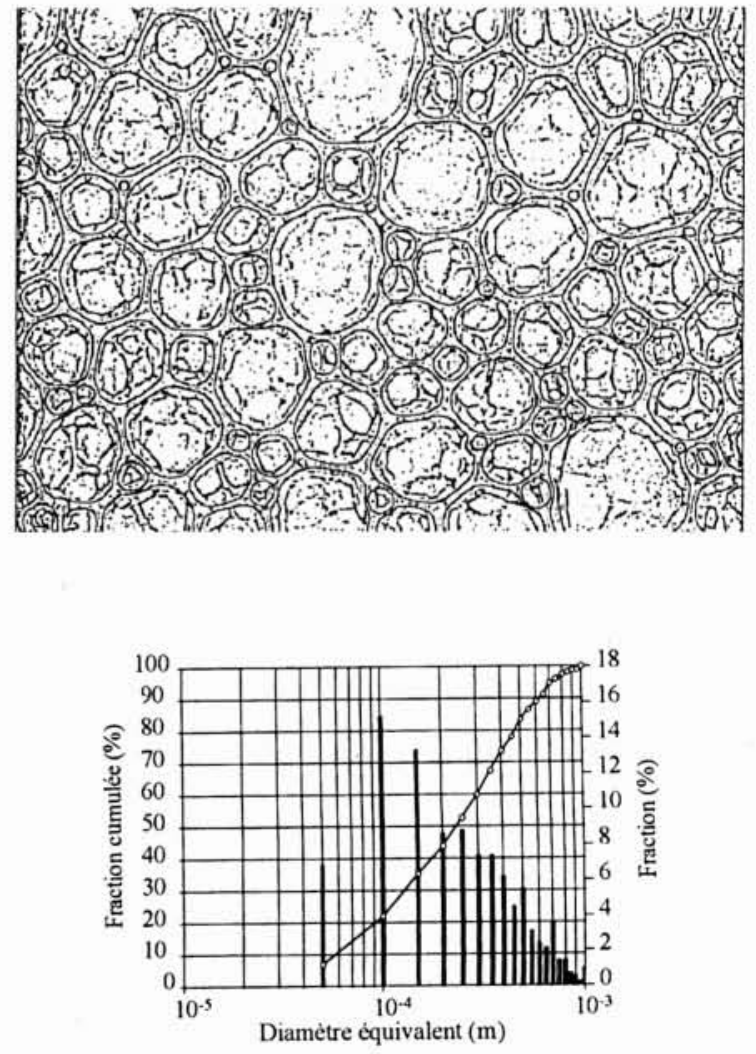

6. (a) image d'origine, (b) image traitée et seuillée, (c) image étiquetée, prête pour les mesures, (d) distribution des tailles de bulles 


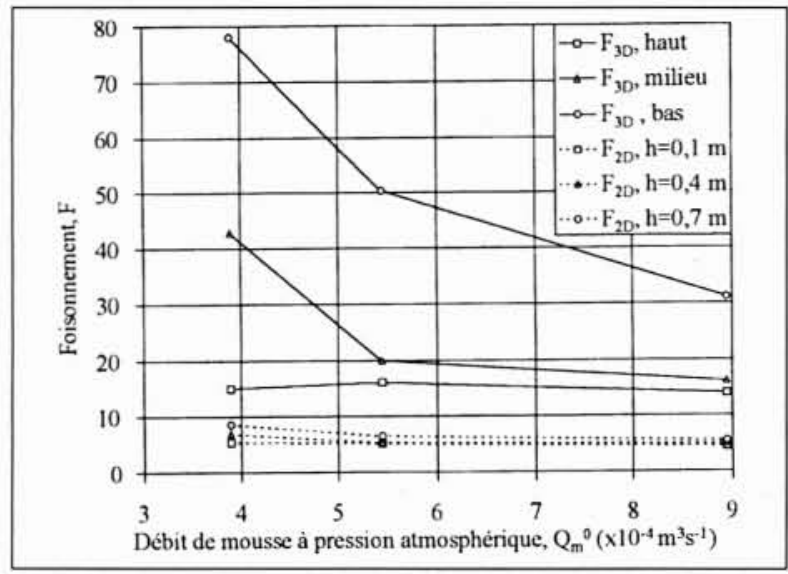

7. $F_{3 D}$ et $F_{2 D}$ en fonction de la hauteur et du débit de mousse

paroi sont prépondérants, même s'il est délicat de relier l'information volumique et l'information surfacique de manière rigoureuse. En particulier, les phénomènes de mouillage et le film liquide en paroi peuvent contribuer à une modification de la structure en paroi.

\subsection{Conclusion}

Une étude simple reposant sur la prise d'échantillons et la mesure du débit de drainage cumulé en fond de colonne a permis de mettre en lumière quelques phénomènes relatifs à la stabilité de la mousse, ainsi que l'influence du débit de mousse. Nous avons également démontré l'utilité de l'étude de la structure par analyse d'images qui nous a permis de formuler l'hypothèse d'une structure de la mousse plus uniforme en paroi que dans le volume de la mousse en raison des phénomènes de mouillage et de la présence du film. Une étude générale est en cours pour connaître l'influence du débit de mousse sur la stabilité dans le but de déterminer le temps de passage optimal de la mousse durant une opération de décontamination.

\section{IV — PERSPECTIVES ET BESOINS}

\subsection{Hydrodynamique}

Grâce aux méthodes de mesure disponibles et aux modèles actuellement développés, nous sommes capables de caractériser l'hydrodynamique d'une mousse de décontamination. Afin d'approfondir les connaissances en la matière, il serait souhaitable de posséder une méthode de mesure physique de l'épaisseur du film en paroi et de la vitesse de glissement. A l'heure actuelle, aucune piste n'a été explorée dans ce sens et seules des estimations sont disponibles. Des recherches pourraient être entreprises en s'orientant vers de mesures ultrasonores, par effet Doppler ou par conductimétrie.

Mesurer le débit volumique de mousse serait également d'un grand intérêt. Actuellement, il n'existe aucune méthode de référence. La valeur élevée $(0,8-0,9)$ de la fraction volumique de gaz semble représenter l'obstacle majeur à la mise au point d'une méthode de mesure directe et en continu. De plus, la structure de la mousse ayant une influence sur son comportement, celle-ci ne doit pas être modifiée lors du passage à travers le débitmètre. Une étude est en cours pour mesurer la vitesse d'écoulement de la mousse dans une tuyauterie grâce à un débitmètre à palettes. Une roue à aubes est entraînée par le passage de la mousse et sa fréquence de rotation est alors proportionnelle à la vitesse d'écoulement du fluide. L'utilisation d'un débitmètre électromagnétique pourrait également se révéler efficace s'il n'engendre pas de compression de la mousse par réduction du diamètre de la conduite.

\subsection{Modèles de drainage}

Concernant l'ensemble des modèles et des mesures de drainage, la discrimination entre la proportion de liquide ruisselant sur la paroi et celle s'écoulant dans le volume est actuellement impossible. Chaque auteur formule donc l'hypothèse que la quantité de liquide est homogène sur l'ensemble de la section étudiée. D'après les résultats obtenus par analyse d'images, cette hypothèse semble réductrice si on veut tenir compte des phénomènes de mouillage en paroi. Un réflexion sur l'étude du ruissellement du liquide en paroi doit donc être menée.

\subsection{Mesure de la fraction gazeuse et de la fraction liquide en continu}

La mesure en continu de la fraction volumique de gaz est primordiale. Cette mesure intéresse aussi bien l'étude de l'écoulement d'une mousse que l'étude de leur stabilité et du drainage. Bien qu'une mesure conductimétrique semble délicate du fait des valeurs élevées de cette fraction volumique $(0,8-0,9)$, elle a été réalisée par Verbist et al. [17] sur une mousse statique. Les résultats restent à confirmer en écoulement, particulièrement pour des vitesses de circulation élevées. Notons également que nous ne pouvons pas estimer sa validité dans le cas où une hétérogénéité de la conductivité intervient (due à un gradient de concentration des réactifs possédant une force ionique importante ou de tensioactifs). Une mesure capacitive pourrait être envisagée, mais aucun travail n'a été réalisé sur le sujet et la présence de liquide comme phase continue pourrait constituer un pont conducteur, limitant ainsi la validité de la mesure. Enfin, Durian et al. [19] proposent une méthode de mesure par diffusion de la lumière qui semblerait pouvoir s'adapter à des mousses s'écoulant à vitesses élevées.

\section{$\mathrm{V} \square \operatorname{CONCLUSION}$}

Deux études séparées ont été menées pour caractériser l'hydrodynamique, la structure et la stabilité des mousses de décontamination. Elles permettent de balayer une large plage de conditions expérimentales. D'une part, un modèle d'écoulement a été proposé et les constantes de ce modèle ont été déterminées. D'autre part, l'étude de la structure et de la stabilité a permis de formuler l'hypothèse que les phénomènes de paroi jouent un rôle prépondérant. Des essais sont en cours pour caractériser le film de glissement (épaisseur, vitesse) et pour relier la structure volumique, la structure en paroi et le drainage.

Au cours de chacune des études, des besoins en moyens de mesure et pour la construction ou l'amélioration de modèles ont été mis à jour. La mesure de l'épaisseur du film et de la vitesse de glissement, ainsi que la mesure du débit volumique de mousse en continu restent à développer. Enfin, les méthodes récentes de mesure de la fraction volumique, ainsi que les modèles de drainage des mousses méritent d'être approfondis.

\section{Références}

[1] FAURY M.I., GAuChON J.P. (1994) - «Decontamination of large volume nuclear components using foams», Contrat CCE $n^{\circ}$ FI2D-0035, (1994) 
[2] Thondavadi N.N., R. Lemlich (1994). - «Flow properties of foam with and without solid paricles», Ind. Eng. Chem. Process Des. Dev., 24, N 3, 748-753, (1985).

[3] Mooney M. (1931). - J. Rheol, (Easton Pa.), 2, [4].

[4] WinkLer W., P. Valko, M.J. Economides. (1994). - «Laminar and drag-reduced polymeric foam flow», J. Rheol., 38, 111-127.

[5] KRAYNIK A.M. (1982), - «Aqueous foam rheology», Annual Meeting of the society of rheology,, paper B10,,

[6] Kroezen A.B.J., J. Groot Wassink, C.A.C. Schipper. (1988). - «The flow properties of foam», JSDC., 108, 393-400,

[7] Heller J. P., M.S. Kuntamukkula. (1987). - «Critical review of the foam rheology literature», Ind. Eng. Chem. Res., 26, 318-325,

[8] HiRasaki G.J., J.B. Lawson. (1983). - Annual Technical Conference and Exhibition of the society of Petroleum Engineers.,, paper SPE 12129.

[9] Calvert J.R. (1991), - «Pressure drop in foam flow», Proc. Inst. Mech. Eng., IMechE Conf., 8, 153-158.

[10] CALvert J.R. (1990). — «Pressure drop for foam flow through pipes», Int. J. Heat \& Fluid Flow, 11, 236-241.

[11] VALKo P., M.J. ECONOMides, (1992). — «Volume equalized constitutive equations for foamed polymer solution", J. Rheol., $36,1033-1055$.
[12] Calvert J.R., K. Nezhati. (1987). - «Bubble size effect in foams», Int. J. Heat \& Fluid Flow, 8, 102-106.

[13] Cheng H.C., R. Lemlich. (1983), - «Errors in the measurement of bubble size distribution in foam», Ind. Eng. Chem. Fundam., 22, 105-109.

[14] Kroezen A.B.J., J. Groot Wassink. (1987). — «Bubble size distribution and energy dissipation in foam mixers», JSDC, 103, 386-394.

[15] Gido S.P., D.E. HiRT, S.M. Montgomery, R.K. Prud'home, L. RenBenfeld. (1989). - « Foam bubble size measured using image analysis before and after passage through a porous medium», J. Dispersion Science and Technology, 10, 785-793.

[16] Guillerme C., W. Loisel, D. Bertrand, Y. Popineau. (1993). - «Study of foam stability by video image analysis : relationship with the quantity of liquid in the foam», Journal of Texture Studies, 24, 287-302.

[17] Verbist G., D. Weaire, A.M. Kraynik. (1996). - «The foam drainage equation», J. Phys. : Condes. Matter., 8, 3715-3731.

[18] Lachaise J., S. Sahnoun, C. Dicharry, B. Mendiboure, J.L. SALAGER. (1991). - «Improved determination of the initial structure of liquid foams», Progr. Colloid. Polym. Sci., 84, 253256.

[19] Durian D.J., D.A. Weitz, D.J. Pine. (1991), - «Multiple light-scattering probes of foam structure and dynamics», Science, 252, 686-688. 